\title{
A treatment planning method to avoid the larynx and eliminate the match-line in the treatment of head and neck cancer with intensity-modulated radiation therapy
}

\author{
Ping Xia • Peng Qi • James Rembert • Andy Z. Hu • \\ Jeanne M. Quivey $\cdot$ Sue S. Yom
}

Received: 13 January 2012 / Accepted: 16 February 2012 /Published online: 9 March 2012

(C) Springer-Verlag 2012

\begin{abstract}
Purpose This study aims to investigate a new planning method that avoids the match-line while maintaining larynx protection as in split field intensity-modulated radiation therapy (IMRT) and to evaluate the potential match-line dose variations with split IMRT.

Methods/materials The new planning method, referred to as selective extended-field IMRT, selected anterior and anterior oblique fields to treat the entirety tumor volumes while restricting other IMRT fields from treating the tumor volumes below the larynx. Five patients with nasopharyngeal cancer, who underwent conventional extended-field IMRT, were replanned using the selective extended IMRT and split-field IMRT techniques.

Results When treatment goals to the target volumes were met, selective extended-field IMRT resulted in an average mean larynx dose of $28.6 \pm 1.4 \mathrm{~Gy}$, as compared with $41.9 \pm 10.6$ and $29.3 \pm 5.6$ Gy for extended-field and split-field IMRT, respectively. For other organs at risk, there were no significant dose differences $(p>0.05)$ among three planning methods.

Conclusions Selective extended-field IMRT achieves comparable tumor volume coverage as the conventional extendedfield IMRT and comparable larynx sparing as split-field IMRT while eliminating field matching.
\end{abstract}

\footnotetext{
P. Xia $(\bowtie) \cdot$ P. Qi

Department of Radiation Oncology, Cleveland Clinic,

Cleveland, OH 44195, USA

e-mail: xiap@ccf.org

P. Xia $\cdot$ P. Qi $\cdot$ J. Rembert • A. Z. Hu • J. M. Quivey $\cdot$ S. S. Yom

Department of Radiation Oncology,

University of California San Francisco,

San Francisco, CA 94143, USA
}

Keywords Intensity modulated radiotherapy $\cdot$ Head and neck cancer $\cdot$ Matchline $\cdot$ Larynx dose reduction $\cdot$ Dysphagia

\section{Introduction}

The treatment of head and neck malignancies poses a continuing technical challenge. The desire to spare normal structures by developing highly conformal dose distributions to the target volume has kept head and neck radiotherapy at the forefront of treatment planning innovation. Over the past decade, IMRT has been rapidly implemented in both academic and community practices, but a consensus has yet to be reached regarding its optimal application.

One debate centers on whether IMRT should be used to cover the entire treatment volume [1-3] or be used for the primary tumor while treating non-adjacent nodal regions with conventional fields. Two typical IMRT techniques are commonly employed. One technique uses beam-split (half-beam blocked) IMRT fields matched with beam-split conventional fields. The second technique, extended-field IMRT (EF-IMRT), uses IMRT fields to treat the entire treatment volume. Although the EF-IMRT technique eliminates the need for field junctions, one of the most widely voiced concerns about this technique is the increased dose to the larynx, which may result in higher levels of dysphagia $[1,4,5]$.

To overcome the clinical and technical limitations of EFIMRT and split-field IMRT (SF-IMRT) planning techniques, we propose a new planning technique, referred to as selective extended-field (SEF)-IMRT. The SEF-IMRT plans utilize typical beam angles from EF-IMRT plans. To effectively protect the larynx while covering the gross lymph nodes located at the anterior aspect of the lower neck, the SEF-IMRT only allows the anterior and anterior oblique fields to treat the entire tumor 
volume while other fields are physically restrained from treating the tumor volume inferior to the larynx.

The first aim of this article is to describe in detail the proposed new planning method and to compare this method with two other typical IMRT methods. The second aim is to evaluate the dosimetric uncertainties generated in matching conventional and IMRT fields as is required in SF-IMRT.

\section{Materials and methods}

Patient selection and dose prescriptions

Five nasopharyngeal patients with primary tumors and associated neck disease extending inferiorly to the larynx were chosen for this study. These patients all were treated with EFIMRT in the University of California San Francisco. Patients were simulated with the neck in a neutral position and were immobilized with a thermoplastic mask fitted to the head and shoulders. The planning gross tumors (PTV-GTV) and planning clinical volumes as either PTV-CTV1 or PTV-CTV2 were contoured by the treating physician. CTV1 generally represents areas adjacent to the GTV at risk for microscopic extension and those nodal regions which are at the highest risk for tumor involvement. At the clinician's discretion, CTV2 were deemed to be at less risk than the CTV1 regions, thus allowing a lower total dose. The following structures were typically contoured during plan optimization: temporal lobes, brainstem, spinal cord, optic chiasm, orbits, lens, optic nerves, parotid glands, maxilla, mandible, temporomandibular joints, tongue (or oral cavity), larynx, trachea, esophagus, and upper lobes of lung.

The treatment goal was to concurrently deliver $70 \mathrm{~Gy}$ to $95 \%$ of the PTV-GTV, 59.4 Gy to $95 \%$ of the PTV-CTV1, and, if applicable, 54.0 Gy to $95 \%$ of the PTV-CTV2 in 33 fractions. Other primary plan acceptance criteria included: maximum dose $<45$ Gy to the spinal cord, $<54$ Gy to the brain stem, mean dose $<26$ Gy to the parotid glands, and $<50$ Gy to the inner ears. Based on our clinical experience, we typically accepted a mean dose $<35-45$ Gy for the larynx in EF-IMRT plans, which was deemed as reasonably achievable without other compromises.

\section{Extended-field IMRT}

The extended-field IMRT (EF-IMRT) technique utilized multiple IMRT fields to treat the primary tumor and all lymph node groups extending into the supraclavicular region. A detailed description of this planning method, including beam angle arrangements and planning dose constraints, was published previously [6]. Typically, nine beam angles were used with a total of 60 segments.

\section{Selective extended-field IMRT}

The selective extended-field IMRT (SEF-IMRT) technique is a modification of EF-IMRT technique, allowing only selected fields to treat the entire set of planning volumes including the lymph nodes in the lower neck and supraclavicular fossa. Because these lymph nodes are located anteriorly, we only allowed anterior and anterior oblique fields to expose the entire tumor volume. The inferior borders of all of the other fields were physically restricted to the regions superior to the larynx.

The treatment planning system (TPS) (Pinnacle V.8.0, Phillips, WI) in active clinical use does not allow users to specify the field border of each beam. Thus, to implement this new planning technique, we used another commercial planning system (Panther V.4.6, Prowess, Concord, CA) to test this planning method. With the Panther TPS, users can draw a restricted exposure region on the beam's eye view to manually define the field border. In addition to restricting non-selected beams from exposing to the larynx, a set of strict planning dose constraints for achieving a mean dose less than 29 Gy were also used to further protect the larynx during IMRT optimization.

For the purposes of this study, the planning CT images and target volume contours were transferred from the Pinnacle system via DICOM protocol to the Panther system. Compared to the clinically deployed EF-IMRT plans, the iso-centers for the SEF-IMRT plans were shifted longitudinally and placed above the larynx. Beam angles and energies in the SEF-IMRT plans were kept the same as those in the EF-IMRT plans. However, the collimator angles were set to $90^{\circ}$ instead of $0^{\circ}$. Except for the larynx, each SEF-IMRT plan was created using the same planning objectives as in the EF-IMRT plan.

\section{Split-field IMRT}

For comparison, SF-IMRT plans were created for all patients using the Pinnacle planning system. The most common SFIMRT technique utilized a single iso-center with half beams of IMRT fields matched to a half beam of an anterior-posterior (AP) supraclavicular field. A single iso-center was placed at the level of the larynx. Multiple half-beam IMRT fields were used to treat the superior portion of the tumor volume. A single half-beam blocked anterior supraclavicular field was used to treat the inferior portion of the tumor volume. The IMRT fields and the anterior supraclavicular fields were matched on the plane of the beam axis at the iso-center. A midline cord block was placed in the anterior supraclavicular field to protect the larynx and the spinal cord.

For fair comparison, the SF-IMRT plans in this study stemmed from the EF-IMRT plans. First, we moved the isocenter of the EF plans to the location (superior of the larynx) where the iso-centers of the SF plans were often located, and 
new EF-IMRT plans with the similar plan quality as the original EF-IMRT plans were created. Then, for each segment of the EF plans, we manually closed all MLC leaves or jaws located below the new iso-center. Finally, we added the anterior supraclavicular (AP) field matched on the plane of the beam axis at the new iso-center. A midline cord block (with a block length cover the AP field length) was placed in the AP field to protect the larynx and spinal cord. The modified EFplan and the AP field were combined as a composite SF-plan for comparison.

Simulation of dose variation at the match-lines of SF plans

To quantitatively evaluate the potential dose variations at the match-lines if a systematic error (either gap or overlap of fields) occurred due to imprecise jaw calibration, we deliberately shifted the upper jaw in the AP field $\pm 1, \pm 2$, and $\pm 3 \mathrm{~mm}$ in the SF-IMRT plans. Furthermore, in order to specifically analyze the dosimetric impact on the pertinent tumor volume and sensitive structures, we renamed PTV-CTV1 $1.5 \mathrm{~cm}$ around the match-line as mPTV_CTV1, and renamed the spinal cord within the same region as m-cord. We used the dose to $95 \%$ of these tumor volumes (D95) and the dose to $1 \mathrm{cc}$ of the m-cord volume as endpoints for match-line dose uncertainty analysis.

Plan comparison and evaluation

To eliminate dose calculation differences between the two treatment planning systems, each SEF-IMRT plan, created from the Panther TPS, was transferred to the Pinnacle TPS for recalculation while keeping segment shapes and monitor units (MUs) the same as in the Panther plan. These plans, referred to as recalculated SEF-IMRT plans, were compared with the original SEF-IMRT plans to confirm the similarity between the two dose calculation methods.

\section{Plan evaluations}

All plans were evaluated based on selected endpoints, such as dose to $95 \%$ of the PTV-GTV, PTV-CTV1, and PTV-CTV2 (D95), maximum dose to the spinal cord, brain stem, temporal lobe, and mandible, and mean dose to the parotid glands, larynx, and inner ears. The Student's $t$ test was used for descriptive statistical analysis. Furthermore, the general plan qualities were compared based upon the homogeneous index as defined in Eq. 1, and the conformity index as defined in Eq. 2. The dose homogeneity index (HI) was defined as the ratio of the maximum point dose and the highest prescription dose

$\mathrm{HI}=D_{\mathrm{Rx}} / D_{\max }$
The plan conformality index (COIN) [7] defined as

$\mathrm{COIN}=\left(\mathrm{PTV}_{\text {ref }} / \mathrm{PTV}\right) \times\left(\mathrm{PTV}_{\text {ref }} /\right.$ Tissue $\left._{\text {ref }}\right)$,

where PTV $\mathrm{ref}_{\text {ref }}$ was the volume of the portion of PTV enclosed by the prescription isodose line and Tissue $_{\text {ref }}$ was the total tissue volume enclosed by the prescription isodose line. The higher HI, the less uniform the plan. The closer COIN was to unity, the more conformal the plan.

\section{Results}

Comparing original SEF and recalculated SEF plans

For all five patients, the first two columns in Table 1 list the mean values and standard deviations of selected endpoints of the original SEF-IMRT plans from the Panther planning system and the recalculated SEF-IMRT plans using the Pinnacle planning system. There are no significant dose differences between the SEF plans and the recalculated SEF plans ( $p=0.77)$. In the rest of this paper, for simplicity, we only compare the recalculated SEF plans with EF and SF plans, and we refer to the recalculated SEF plans as SEF plans.

\section{Comparisons of three types of IMRT plans}

The detailed dosimetric comparison of selected endpoints of the SEF-, EF-, and SF-IMRT plans are shown in Figs. 1, 2, and 3 . The average values and the standard deviation of these endpoints are listed in Table 1. As shown from Table 1, when compared to the EF plans, the SEF- and SF-IMRT plans achieved a significant $(p=0.017)$ dose reduction to the larynx with average mean dose to the larynx of $28.6 \pm 1.4$ and $29.3 \pm$ $5.6 \mathrm{~Gy}$, respectively, compared to $41.9 \pm 10.6 \mathrm{~Gy}$ in the EFIMRT plans. More specifically, as shown in Fig. 1, the mean larynx dose in SEF plans was more consistent than that of in EF plans, with a standard deviation of 1.4 Gy in SEF plans compared to 10.6 Gy in EF plans.

For the tumor volumes, when SEF plans delivered the goal prescription doses to the PTV-GTV and PTV-CTV2, the average D95 of the PTV-CTV1 was within $96 \%$ of the prescription dose. In comparison, the average D95 from the EF plans achieved $100 \%$ of the prescription dose. For SF plans, the average D95s of PTV-GTV, PTV-CTV1, and PTV-CTV2 were $96 \%, 93 \%, 91 \%$ of the prescription doses. The detailed D95s of PTV-GTV and PTV-CTV1 for all patients among these plans are shown in Fig. 2a-b. Because not all patients had PTV-CTV2, the plot of D95 of PTV-CTV2 is omitted. For patients with enlarged lymph nodes in the lower neck and supraclavicular regions, the dose coverage to PTV-CTV1 and PTV-CTV2 may have been compromised with the SF plans. 
Table 1 Comparisons of the original and recalculated SEF-IMRT, SF-, and EF-IMRT plans in terms of target coverage and dose to critical structures (all numbers are in the unit of Gy)
$D_{95}$ dose to $95 \%$ of the target volume; $D_{\max }$ maximum dose; $D_{\text {mean }}$ mean dose

\begin{tabular}{lcccc}
\hline Structures $($ dose $)$ & Original SEF-IMRT & Recalculated SEF-IMRT & SF-IMRT & EF-IMRT \\
\hline PTV-GTV $\left(D_{95}\right)$ & $71.1 \pm 0.9$ & $70.6 \pm 1.6$ & $67.1 \pm 6.6$ & $72.7 \pm 1.6$ \\
PTV-CTV1 $\left(D_{95}\right)$ & $58.7 \pm 0.6$ & $57.2 \pm 1.5$ & $55.2 \pm 3.4$ & $59.9 \pm 1.5$ \\
PTV-CTV2 $\left(D_{95}\right)$ & $54.7 \pm 0.5$ & $52.6 \pm 1.4$ & $48.9 \pm 9.6$ & $57.2 \pm 1.7$ \\
Larynx $\left(D_{\text {mean }}\right)$ & $29.4 \pm 3.0$ & $28.6 \pm 1.4$ & $29.3 \pm 5.6$ & $41.9 \pm 10.6$ \\
Spinal cord $\left(D_{\text {max }}\right)$ & $41.9 \pm 2.6$ & $41.9 \pm 2.2$ & $45.5 \pm 3.7$ & $45.6 \pm 3.7$ \\
Brain stem $\left(D_{\text {max }}\right)$ & $54.3 \pm 4.0$ & $53.4 \pm 4.5$ & $56.2 \pm 6.7$ & $55.8 \pm 6.2$ \\
Mandible $\left(D_{\text {max }}\right)$ & $70.3 \pm 1.6$ & $69.0 \pm 2.0$ & $71.6 \pm 2.7$ & $71.4 \pm 2.3$ \\
Temporal lobe $\left(D_{\text {max }}\right)$ & $63.3 \pm 4.9$ & $65.3 \pm 7.0$ & $67.0 \pm 9.4$ & $66.4 \pm 9.4$ \\
Left parotid $\left(D_{\text {mean }}\right)$ & $27.9 \pm 1.7$ & $27.4 \pm 2.1$ & $28.3 \pm 3.2$ & $28.5 \pm 3.0$ \\
Right parotid $\left(D_{\text {mean }}\right)$ & $27.2 \pm 1.7$ & $26.4 \pm 1.1$ & $28.4 \pm 2.2$ & $28.6 \pm 2.0$ \\
Left inner ear $\left(D_{\text {mean }}\right)$ & $44.9 \pm 6.3$ & $43.4 \pm 7.1$ & $50.4 \pm 12.5$ & $50.4 \pm 12.3$ \\
Right inner ear $\left(D_{\text {mean }}\right)$ & $40.6 \pm 6.2$ & $40.1 \pm 7.6$ & $38.7 \pm 6.3$ & $38.6 \pm 6.3$ \\
\hline
\end{tabular}

Although D95 of the PTV-CTV1 in SEF plans is slightly lower that of in EF plans, the difference was not statistically significant $(p=0.14)$.

Among the three types of plans, no significant differences were found in the mean doses to the parotid glands and inner ears or in the maximum doses to the spinal cord and brain stem $(p=0.55)$, as shown in Table 1 . The detailed maximum doses of the spinal cord and brain stem for each patient were plotted in Fig. 3a, b.

Figure $4 \mathrm{a}, \mathrm{b}$ shows plan homogeneity index and conformal index of the PTV-CTV1 for the three types of plans for each patient. The average plan homogeneity indices were not significantly different $(p=0.97)$ among the three types of plans, with $85.1 \%, 84.5 \%, 84.4 \%$ for SEF, EF, and SF plans. The average plan conformal indices of the PTV-CTV1 were 0.62 , 0.55, and 0.66 for SEF, EF, and SF plans, respectively. We did not include plan homogeneity index and conformal index of other tumor volume because not all patients had PTV-CTV2. For the PTV-GTV, because of different prescription dose than that of PTV-CTV1 with the same the maximum dose of a plan, the plan homogeneity indices for the PTV-GTV would have the same trend as the PTV-CTV1 but with different the

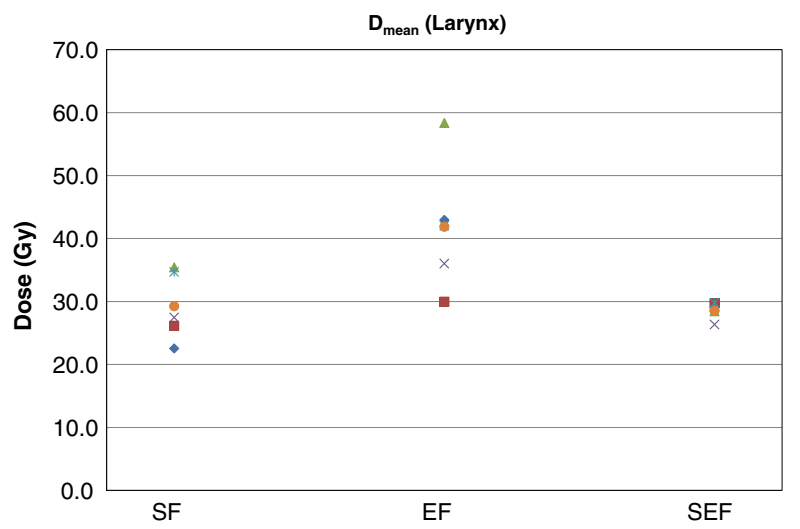

Fig. 1 Comparison of mean dose of the larynx in the SF, EF, and SEF plans absolute values of the homogeneity indices. The conformal indices of the PTV-GTV for these three types of plans are shown in Fig. 4c. The average plan conformal indices of the PTV-GTV were $0.38,0.32$, and 0.39 for the SEF, EF, and SF plans, respectively.

Dose uncertainties near match-line

For SF-IMRT plans, a gap measuring 1, 2, and $3 \mathrm{~mm}$ wide was introduced between the AP field and the IMRT field at
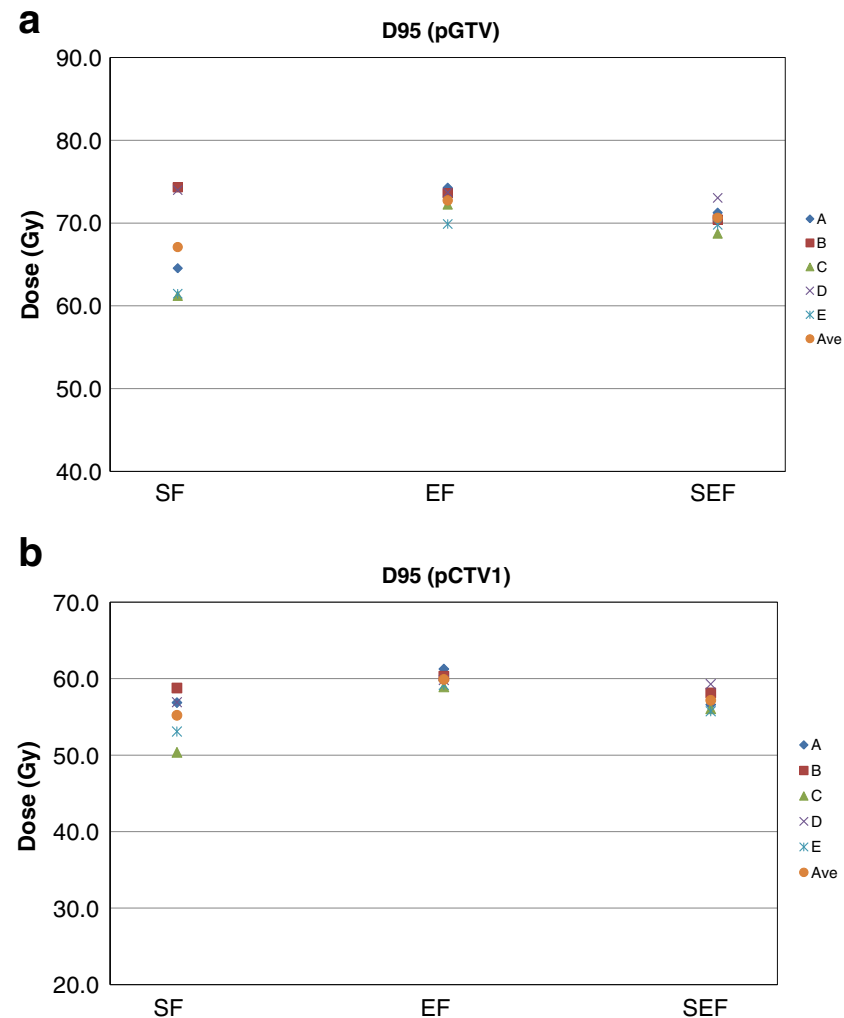

Fig. 2 Comparison of tumor dose coverage of a PTV-GTV and b PTVCTV1 in the SF, EF, and SEF plans 
a

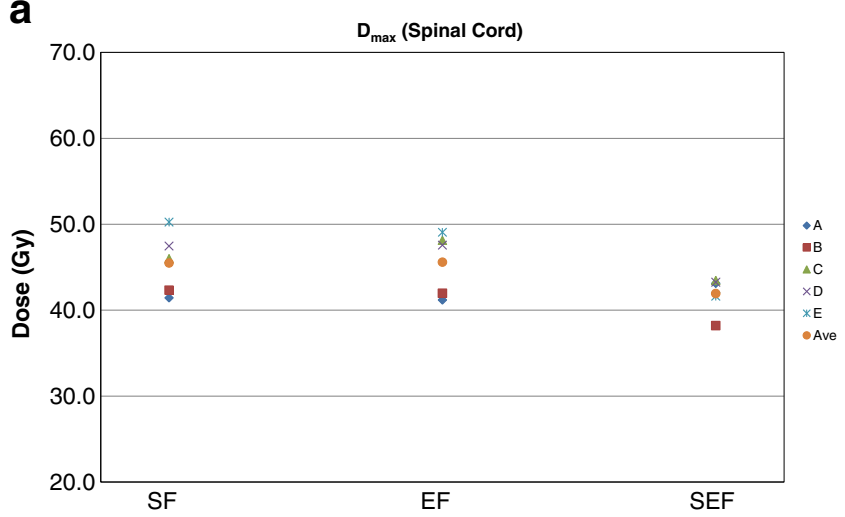

b

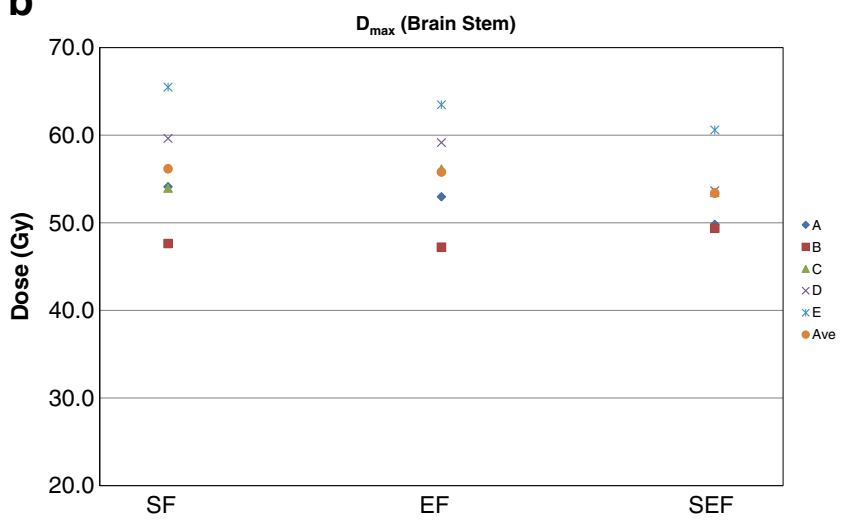

Fig. 3 Comparison of maximum doses to $\mathbf{a}$ the spinal cord and $\mathbf{b}$ the brain stem in the SF, EF, and SEF plans

the match-line. These deliberately introduced errors resulted in an average of $6.0 \pm 4.6 \%, 16.5 \pm 6.1 \%$, and $24.2 \pm 9.1 \%$ reduction in D95 to the $\mathrm{mPTV}-\mathrm{CTV} 1$, respectively. An overlap of 1,2 , and $3 \mathrm{~mm}$ of the AP field and the IMRT field resulted in an increase of $12.3 \pm 11.7 \%, 23.8 \pm 8.0 \%$, and $35.6 \pm 12.8 \%$ in the D1cc dose of the match spinal cord region, respectively. Figure 5 shows the isodose changes with overlaps of $0,1,2$, and $3 \mathrm{~mm}$ between the border of the AP field and IMRT fields.

\section{Discussions}

Compared to a single iso-center half-beam-blocked SF-IMRT technique, SEF-IMRT achieves improved target coverage with similar larynx sparing. Compared to the EF-IMRT technique, SEF-IMRT achieves similar tumor coverage with a substantial reduction of mean dose to the larynx. Similar to SF-IMRT, SEF-IMRT blocks all posterior and lateral beams to the low neck region while minimizing larynx exposure from anterior and anterior oblique beams by incorporating laryngeal sparing into the optimization. The SEF-IMRT technique also eliminates the need for a sharp field junction as in SF-IMRT.

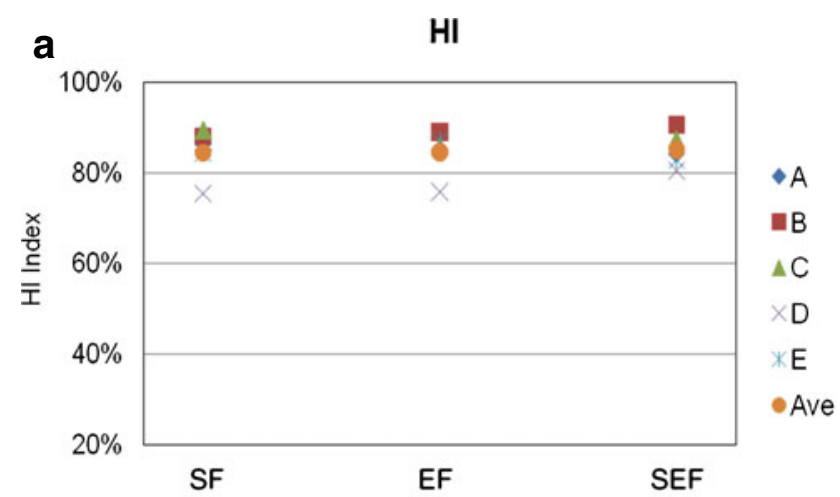

b $\quad \operatorname{coIN}(\mathrm{pCTV} 1)$

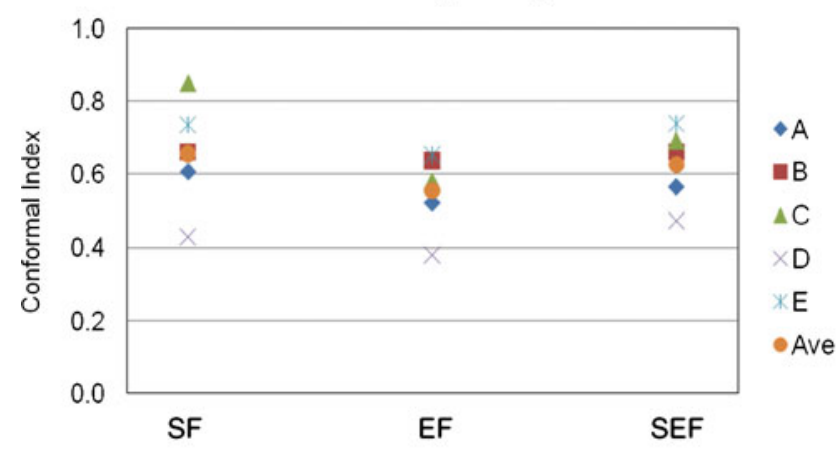

C

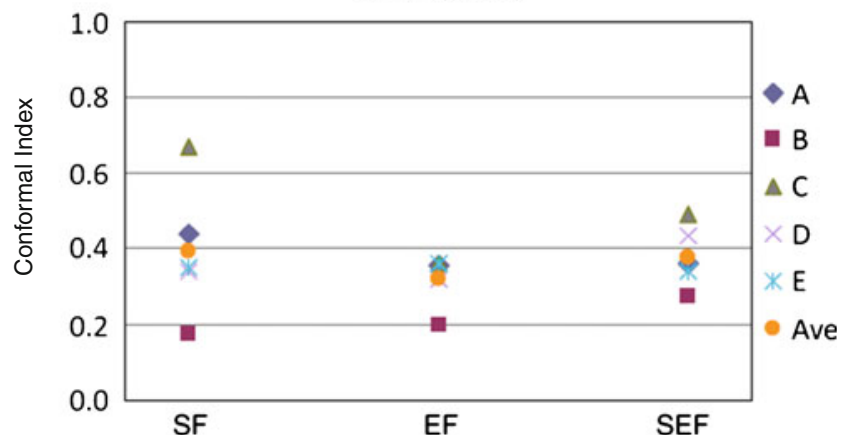

Fig. 4 Comparison of a plan homogeneity indices of the PTV-CTV1, b conformal indices of the PTV-CTV1, and c conformal indices of PTV-GTV in the SF, EF, and SEF plans

The reduction of mean dose to the larynx may have major impact on a patient's quality of life. Fua et al. [8] reported that a mean dose of the larynx of 55.2 Gy resulted in $95 \%$ of grade 3 dysphagia for a group of patients with nasopharyngeal cancer, and a mean dose of the larynx of $27.2 \mathrm{~Gy}$ reduced the proportion of grade 3 dysphagia to $62.5 \%$. Using endpoints of percutaneous endoscopic gastrostomy (PEG) tube dependence at 12 months and aspiration on modified barium swallow testing, Caudell et al. [9] found that a mean dose of the larynx $>41$ Gy and V $60>24 \%$ were clinically significant dosimetric parameters.

Whether to prioritize protection of the larynx or achieve comprehensive tumor coverage in the neck is at the center of 
Fig. 5 Isodose changes in a SF plan with an overlap of $0,1,2$, and $3 \mathrm{~mm}$ between the border of the AP field and IMRT fields. Solid blue is the PTV-GTV, and solid pink is the PTV-CTV1. The isodose lines of $50.4,44.8$, and $42.0 \mathrm{~Gy}$ are displayed in yellow, light blue, and green, respectively
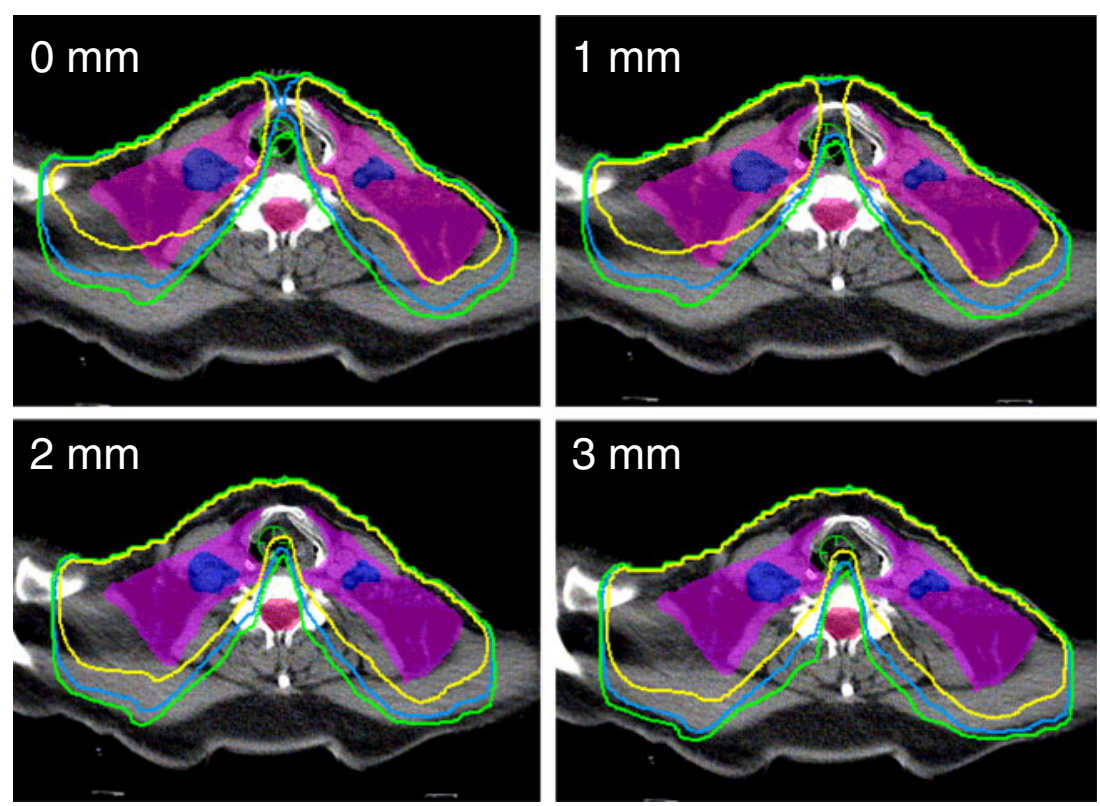

50.4/1.8Gy

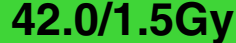

the debate about the advantages and disadvantages of extended-field IMRT and split-field IMRT. Dabaja et al. [2] compared EF-IMRT and SF-IMRT for a group of early-stage oropharyngeal patients and found that acceptable plans can be generated with either EF-IMRT or SF-IMRT except for the issue concerning the mean dose to the larynx. The mean larynx dose was 47 Gy with EF-IMRT compared to 18.7 Gy with SF-IMRT. Therefore, they recommended that SF-IMRT was only preferred for patients with a primary tumor not involving the base of tongue, patients with clinically uninvolved neck nodes, or patients without bulky lymph nodes near the match-line. Otherwise, EF-IMRT was recommended. By comparing the outcomes of 91 patients treated with EF-IMRT and with SF-IMRT, Turaka et al. [10] concluded that for patients with early $\mathrm{T}$ and early $\mathrm{N}$ disease, using the conventional low neck field did not increase the risk of local-regional failure nor decrease PEG tube dependence. They also recommended that when the lymph nodes in the low neck are greater than $3 \mathrm{~cm}$ in size, using matching fields in SF-IMRT should be avoided.

Concerning the dose uncertainties at the match-line in SFIMRT, several feathering techniques have been developed and implemented clinically [11, 12]. A traditional method is to move the match-line (or the iso-center) once or twice during the course of the treatment. However, shifting the match-line between the IMRT plan and the AP field is not as straightforward as shifts might be in 3D conformal plans. Another method is to deliberately generate a wedge type of dose gradient near the matching region [12], creating a gradual dose gradient to minimize potential hot or cold spots in the event of an imperfect match. This method has not been widely adopted clinically.
Sources of match-line dosimetric uncertainty can be attributed to inaccuracies of patient set-up, inadequate immobilization, and imprecise field abutment due to mechanical factors (independent jaw position, gantry/collimator tolerance). Inaccurate patient positioning and inadequate immobilization are major sources of random errors. Mechanical factors, such as the imprecision of the independent jaw, gantry, and collimator placement, are sources of systematic errors. An acceptable $\pm 1 \mathrm{~mm}$ precision for independent jaws may create up to $2 \mathrm{~mm}$ of possible match-line jaw overlap/underlap. This imprecise jaw placement can induce match-line inhomogeneities of $15-20 \% / \mathrm{mm}$ [12-14]. These results are consistent with our findings in this study. But how does this dosimetric uncertainty translate into the coverage of planned treatment volumes and critical structures? Bubenzer et al. [15] found that match-line TLDs located approximately at the spinal cord position received markedly more doses than predicted. In the present study, we specifically evaluated doses to the tumor volume and spinal cord at the region of the match-line.

In general, there is a consensus that because of using multiple beams, the mean larynx dose in EF-IMRT is higher than that of SF-IMRT, even with a strict planning dose constraint applied to the larynx during optimization of EFIMRT. In theory, a robust optimization algorithm would automatically block beams without using the method described in SEF-IMRT. Unfortunately, such robust optimizers are not currently widely available to many clinical users. Reports from other institutions as in references [1-3] further support this consensus. Moreover, SF-IMRT method is still widely used clinically to better protecting the larynx despite of knowing the match-line uncertainties and potential underdose 
to the tumor volumes. There are other practical reasons for SFIMRT. For example, for some linear accelerators (e.g., Varian machine), including the lower neck into the IMRT plans such as in EF-IMR may result in splitting an IMRT field into two and thus prolong the treatment time. With different IMRT delivery mechanisms and more robust optimization, the need of different planning methods such as described in EF-, SEF-, and SF-IMRT methods may disappear because a robust optimizer may automatically select a planning method without user's input. Currently, with limitation in most commercially available IMRT optimization, we believe that SEF-IMRT provides a good compromise solution between EF-IMRT and SF-IMRT techniques.

As in any other retrospective planning comparison study, one of the limitations of this study is that it could not completely eliminate other confounding variables in the compared plans. Due to the limitation of available tools in our clinical planning system (the Pinnacle system), we used two different treatment planning systems, which unavoidably introduced differences in plan optimization. With the current versions of the Pinnacle system (from version 8.0 to 9.0) and with the requirement of secondary jaws following each segment in Siemens linear accelerators, we were not able to produce SEF-IMRT plans using our clinical planning system. Because of different optimization strategies in the two planning systems, we could not guarantee that the planning dose constraints applied in the SEF-IMRT plans the same as those in the EF-IMRT plans, although we used the same plan acceptance criteria such as the dose coverage to the tumor volumes and dose sparing to the other sensitive structures except for the larynx. In the SEF-IMRT plans, we tightened the larynx planning dose constraint, which enabled us to reduce the mean larynx dose without compromising other plan qualities. One may speculate if a strict planning dose constraint were also applied to the larynx in EF-IMRT plans, it might reduce the larynx dose to the same level as the SF-IMRT or SEF-IMRT plans. In our experience, this approach can be very onerous. Other study [2] has previously shown that except for the larynx, EF-IMRT plans are comparable to SF-IMRT plans. We believe the higher larynx dose in EF-IMRT plans is due to the use of seven to nine beams typically seen in EF-IMRT plans. Finally, we did not attempt to further optimize the EFIMRT plans but use the clinically accepted EF-IMRT plans for comparison because we believed that alternation risked losing the special value of clinical plans since these plans underwent careful review by attending radiation oncologists. Thus, these original plans represent a realistic version of what can be clinically achieved using EF-IMRT.

Another limitation of this study is that it is a planning study not yet confirmed by experimental measurements. In addition, the advantages of the SEF IMRT planning method have not been confirmed by clinical outcomes. All of the patients had nasopharyngeal cancer; further study is required to investigate whether the observed advantages of SEF-IMRT can be extended to other head and neck cancers.

\section{Conclusions}

In the treatment of the head-and-neck cancer, there is no consensus on whether and when to use extended-field IMRT or split-field IMRT. To combine the advantages of these two treatment planning techniques, we propose a new IMRT planning method that avoids the field matching of beam-split IMRT but achieves tumor volume coverage comparable to EF-IMRT and larynx sparing comparable to SF-IMRT. Further study is needed to investigate this planning method in the clinical setting.

Conflict of interest This research is supported in part by Siemens Medical Solutions through a research grant.

\section{References}

1. Amdur RJ, Li JG, Liu C, Hinerman RW, Mendenhall WM (2004) Unnecessary laryngeal irradiation in the IMRT era. Head Neck 26 (3):257-263. doi:10.1002/hed.10379, discussion 263-254

2. Dabaja B, Salehpour MR, Rosen I, Tung S, Morrison WH, Ang KK, Garden AS (2005) Intensity-modulated radiation therapy (IMRT) of cancers of the head and neck: comparison of split-field and whole-field techniques. Int J Radiat Oncol Biol Phys 63 (4):1000-1005. doi:10.1016/j.ijrobp.2005.03.069

3. Lee N, Mechalakos J, Puri DR, Hunt M (2007) Choosing an intensity-modulated radiation therapy technique in the treatment of head-and-neck cancer. Int J Radiat Oncol Biol Phys 68 (5):1299-1309. doi:10.1016/j.ijrobp.2006.11.019

4. Feng FY, Kim HM, Lyden TH, Haxer MJ, Worden FP, Feng M, Moyer JS, Prince ME, Carey TE, Wolf GT, Bradford CR, Chepeha DB, Eisbruch A (2010) Intensity-modulated chemoradiotherapy aiming to reduce dysphagia in patients with oropharyngeal cancer: clinical and functional results. J Clin Oncol 28(16):2732-2738. doi:10.1200/JCO.2009.24.6199

5. Gluck I, Feng FY, Lyden T, Haxer M, Worden F, Chepeha DB, Eisbruch A (2009) Evaluating and reporting dysphagia in trials of chemoirradiation for head-and-neck cancer. Int J Radiat Oncol Biol Phys 77(3):727-733. doi:10.1016/j.ijrobp.2009.05.049

6. Ludlum E, Xia P (2008) Comparison of IMRT planning with twostep and one-step optimization: a way to simplify IMRT. Phys Med Biol 53(3):807-821. doi:10.1088/0031-9155/53/3/018

7. Baltas D, Kolotas C, Geramani K, Mould RF, Ioannidis G, Kekchidi M, Zamboglou N (1998) A conformal index (COIN) to evaluate implant quality and dose specification in brachytherapy. Int J Radiat Oncol Biol Phys 40 (2):515-524

8. Fua TF, Corry J, Milner AD, Cramb J, Walsham SF, Peters LJ (2007) Intensity-modulated radiotherapy for nasopharyngeal carcinoma: clinical correlation of dose to the pharyngo-esophageal axis and dysphagia. Int J Radiat Oncol Biol Phys 67(4):976-981. doi:10.1016/j.ijrobp.2006.10.028

9. Caudell JJ, Schaner PE, Desmond RA, Meredith RF, Spencer SA, Bonner JA (2010) Dosimetric factors associated with long-term dysphagia after definitive radiotherapy for squamous cell carcinoma of the head and neck. Int J Radiat Oncol Biol Phys 76(2):403-409. doi:10.1016/j.jirobp.2009.02.017 
10. Turaka A, Li T, Nicolaou N, Lango MN, Burtness B, Horwitz EM, Ridge JA, Feigenberg SJ (2010) Use of a conventional low neck field (LNF) and intensity-modulated radiotherapy (IMRT): no clinical detriment of IMRT to an anterior LNF during the treatment of head-and neck-cancer. Int J Radiat Oncol Biol Phys 79 (1):65-70. doi:10.1016/j.ijrobp.2009. 10.034

11. Amdur RJ, Liu C, Li J, Mendenhall W, Hinerman R (2007) Matching intensity-modulated radiation therapy to an anterior low neck field. Int J Radiat Oncol Biol Phys 69(2 Suppl):S46S48. doi:10.1016/j.ijrobp.2007.04.091

12. Duan J, Shen S, Spencer SA, Ahmed RS, Popple RA, Ye SJ, Brezovich IA (2004) A dynamic supraclavicular field-matching technique for head-and-neck cancer patients treated with IMRT.
Int J Radiat Oncol Biol Phys 60(3):959-972. doi:10.1016/j. ijrobp.2004.06.213

13. Fabrizio PL, McCullough EC, Foote RL (2000) Decreasing the dosimetric effects of misalignment when using a mono-isocentric technique for irradiation of head and neck cancer. Int $\mathrm{J}$ Radiat Oncol Biol Phys 48(5):1623-1634

14. Rosenthal DI, McDonough J, Kassaee A (1998) The effect of independent collimator misalignment on the dosimetry of abutted half-beam blocked fields for the treatment of head and neck cancer. Radiother Oncol 49(3):273-278

15. Bubenzer V, Esfandi M, Jafari N, Manske MA, Wong PF (2004) Dosage along the matchline between upper head-and-neck IMRT fields and conventional supraclavicular fields. Med Dosim 29 (2):104-108. doi:10.1016/j.meddos.2004.03.003 